GRASAS Y ACEITES 65 (3)

July-September 2014, e036

ISSN-L: 0017-3495

doi: http://dx.doi.org/10.3989/gya.0115141

\title{
Fatty acid profile and cholesterol and retinol contents in different locations of Celta pig breed
}

\author{
R. Domínguez, S. Martínez, J. Carballo and I. Franco ${ }^{\bowtie}$ \\ Área de Tecnología de los Alimentos, Facultad de Ciencias, Universidad de Vigo, 32004 Ourense, Spain \\ Corresponding author: inmatec@uvigo.es
}

Submitted: 21 January 2014; Accepted: 28 April 2014

SUMMARY: The fatty acid profile (total, neutral and polar lipids) and cholesterol and retinol contents of the intramuscular -Longissimus dorsi (LD) and Psoas major (PM)-, adipose -rump, covering Biceps femoris muscle, ventral and dorsal-, perirenal and hepatic fat of the Celta pig breed (Galicia, northwest Spain) were evaluated. Oleic acid was the most abundant fatty acid, except in the polar lipids of the intramuscular fat, where the most abundant fatty acid was linoleic acid. The fatty acid composition of total and neutral lipids was influenced more by the location than the fatty acids of polar lipids. There were some differences only in minor fatty acids of intramuscular and subcutaneous fat between castrated females and castrated males. The fatty acids of neutral lipids were also more influenced by sex than the fatty acids of polar lipids. The cholesterol and retinol contents showed no significant differences by sex. The LD muscle showed a higher content of intramuscular fat than the PM muscle. The greatest cholesterol values were described in the liver. Subcutaneous locations showed higher cholesterol levels than muscles.

KEYWORDS: Celta pig; Cholesterol; Fatty acids; Location; Retinol; Sex

RESUMEN: Perfil de ácidos grasos y contenidos de colesterol y retinol en diferentes localizaciones del cerdo de raza Celta. Se estudió el perfil de los ácidos grasos y los contenidos en colesterol y retinol en la grasa intramuscular - Longissimus dorsi (LD) y Psoas mayor (PM) -, subcutánea -grupa, ventral, dorsal y la grasa que rodea al músculo Bíceps femoris -, perirrenal y hepática de cerdos de raza Celta (Galicia, noroeste de España). El ácido oleico fue el ácido graso más importante, excepto en los lípidos polares de la grasa intramuscular, donde el ácido graso más abundante fue el ácido linoleico. La composición de ácidos grasos de los lípidos totales y neutros estuvo más influenciada por la localización grasa que los ácidos grasos de los lípidos polares. Se encontraron ligeras diferencias entre hembras y machos castrados en algunos ácidos grasos minoritarios en la grasa intramuscular y subcutánea. Los ácidos grasos de los lípidos neutros fueron más influenciados por el sexo que los ácidos grasos de los lípidos polares. El contenido en colesterol y retinol no mostró diferencias significativas entre sexos. El músculo LD presentó un mayor contenido de grasa intramuscular que el PM. Los mayores valores de colesterol fueron determinados en el hígado. En las localizaciones subcutáneas los contenidos en colesterol fueron más elevados que en la grasa intramuscular.

PALABRAS CLAVE: Ácidos grasos; Cerdo Celta; Colesterol; Localización; Retinol; Sexo

Citation/Cómo citar este artículo: Domínguez R, Martínez S, Carballo J, Franco I. 2014. Fatty acid profile and cholesterol and retinol contents in different locations of Celta pig breed. Grasas Aceites 65 (3): e036. doi: http://dx.doi. org/10.3989/gya.0115141.

Copyright: (C) 2014 CSIC. This is an open-access article distributed under the terms of the Creative Commons Attribution-Non Commercial (by-nc) Spain 3.0 Licence. 


\section{INTRODUCTION}

The Celta pig is an autochthonous porcine breed raised traditionally in Galicia (northwest of Spain). Because of their poor growth rate, carcass conformation and crossbreeding between local pigs and improved breeds, the Celta breed became extinct during the second half of the 20th century. At the present time, their population is part of a project of recuperation, conservation, and promotion of this breed backed by the Autonomous Regional Government of Galicia (Carril et al., 2001).

The aim of the recovery of the Celta pig breed is not only to obtain high-quality meat, but also to contribute to the environmental preservation and the recovery of traditional practices. Celta pigs can be reared in an intensive, semi-intensive or freerange system. The use of local breed and extensive or semi-extensive feeding systems based on natural feed resources (chestnuts, grass, etc.) could also help to maintain development in the rural populations of northwestern Spain. On the other hand, the recovery of the Celta pig breed also has the objective the producing high-quality dry-cured meat products such as "lacón" (Lorenzo et al., 2014), dry-cured ham (Bermúdez et al., 2012; 2014; Lorenzo et al., 2013) and "chorizo" (Gómez and Lorenzo, 2013).

The carcass quality and meat quality are determined by factors such as breed, sex, rearing system, age at slaughter or physical exercise. Among the compositional traits related to meat quality, the fat is known to be very important. Fat contributes to the texture, flavor and juiciness of the meat. The fatty acid content and its types determine the physical and nutritional characteristics of fat, and they are associated with human health which is why they are of such great interest (Václavková and Bečková, 2007; Wood et al., 2004).

Many studies have reported that the constitutive fatty acids of the fat are influenced by factors such as genotype (Gandemer et al., 1992), types and percentages of feeding (García-Olmo et al., 2002; Rentfrow et al., 2003), age at slaughtering (Bragagnolo and Rodríguez-Amaya, 2002), location in the carcass (Delgado et al., 2002; Vázquez et al., 1996), sex and castration (Cordero et al., 2010; Wood et al., 1989) or slaughter weight (Palanska et al., 1993). However, there are few studies that compare the effects of the anatomical location and sex on fatty acids, cholesterol, retinol and fat contents of the carcass of castrated females and castrated males.

The aim of this research was to study the fatty acid profile (total lipids, neutral lipids and polar lipids), cholesterol and retinol contents of the fat of the Celta pig breed of eight fat deposits in castrated females and castrated males.

\section{MATERIALS AND METHODS}

\subsection{Pigs, samples and diet}

In order to carry out this study, 12 castrated Celta pigs ( 6 males and 6 females) were continuously fed with commercial compound feed. The piglets, which were vaccinated and deparasitized following the usual protocols, were suckled until an age of 40 days. The males were castrated at 75 days of age and the females were castrated at 90 days of age. All pigs were reared and fattened until the age of 16 months in an extensive regime, with a livestock density of 12 animals per hectare.

After slaughtering, and after 24 hours of refrigeration, samples of intramuscular fat (Longissimus dorsi and Psoas major muscles), subcutaneous fat (rump, subcutaneous ventral, subcutaneous dorsal and covering of the Biceps femoris muscle), perirenal fat and liver fat were obtained from each carcass.

The chemical composition, retinol and cholesterol contents and fatty acid composition of the diet are shown in Table 1. The chemical composition analysis of commercial compound feed was carried out according the methods of A.O.A.C. (2000) and fatty acids, retinol and cholesterol analysis were performed according to the methods described below.

\subsection{Fat and fatty acid analyses}

The intramuscular and hepatic fats were extracted following the procedure described by Folch et al. (1957) and the subcutaneous and perirenal fats were extracted following the procedure described by De Pedro et al. (1997).

In order to determine if there are significant differences between the two methods of fat extraction, fat was extracted in triplicate from two different locations with both methods, and the lipid profile was analyzed. There were no significant differences $(\mathrm{P}<0.05)$ in the fatty acid profile between the two extraction methods.

Intramuscular fat content (IMF) was quantified according to the ISO 1443:1979.

The neutral and polar lipids from muscles, perirenal, subcutaneous and liver samples were obtained according to the procedure developed by Kaluzny et al. (1985). Fat extracts were methylated and the fatty acid profile of both lipids were determined using the procedure described by Franco et al. (2006).

Fatty acid methyl esters were analyzed by Gas Chromatography using a Thermo Finnigan Trace GC (Thermo Finnigan, Austin, TX, USA). The separation of the different fatty acids was carried out in an Innowax column: $30 \mathrm{~m} ; 0.25 \mathrm{~mm}$ ID; $0.25 \mu \mathrm{m}$ film thickness (Agilent Technologies, Palo Alto, CA, USA). The temperature of the detector was $250^{\circ} \mathrm{C}$ and that of the injector $230^{\circ} \mathrm{C}$. The chromatographic conditions used in the determination of 
TABLE 1. Chemical composition (expressed as $\mathrm{g} \cdot 100 \mathrm{~g}^{-1}$ ) and fatty acids of commercial compound feed

\begin{tabular}{|c|c|}
\hline Moisture & 10.5 \\
\hline Crude protein & 15.3 \\
\hline Eter extract & 3.9 \\
\hline Crude fiber & 4.6 \\
\hline Starch & 39.7 \\
\hline Ash & 6.3 \\
\hline Cholesterol (mg·100 g $\left.\mathrm{g}^{-1}\right)$ & 32.3 \\
\hline Retinol $\left(\mu \mathrm{g} \cdot 100 \mathrm{~g}^{-1}\right)$ & 180 \\
\hline \multicolumn{2}{|l|}{ Fatty acids } \\
\hline $\mathrm{C} 12: 0$ & $5.56 \pm 0.06$ \\
\hline C14:0 & $41.70 \pm 0.19$ \\
\hline $\mathrm{C} 14: 1$ & $0.17 \pm 0.02$ \\
\hline $\mathrm{C} 15: 0$ & $4.87 \pm 0.00$ \\
\hline $\mathrm{C} 15: 1$ & $0.97 \pm 0.03$ \\
\hline $\mathrm{C} 16: 0$ & $873.46 \pm 0.37$ \\
\hline $\mathrm{C} 16: 1 n 7$ & $57.13 \pm 0.26$ \\
\hline $\mathrm{C} 17: 0$ & $12.98 \pm 0.24$ \\
\hline $\mathrm{C} 17: 1$ & $7.19 \pm 0.08$ \\
\hline $\mathrm{C} 18: 0$ & $361.43 \pm 0.08$ \\
\hline $\mathrm{C} 18: 1$ c $n 9$ & $1048.48 \pm 0.57$ \\
\hline $\mathrm{C} 18: 2$ n6 & $1012.74 \pm 3.37$ \\
\hline $\mathrm{C} 18: 3 n 6$ & $2.12 \pm 0.08$ \\
\hline $\mathrm{C} 18: 3 n 3$ & $97.54 \pm 0.24$ \\
\hline $\mathrm{C} 20: 0$ & $4.98 \pm 0.04$ \\
\hline $\mathrm{C} 20: 1 n 9$ & $15.11 \pm 0.09$ \\
\hline $\mathrm{C} 20: 2 n 6$ & $5.10 \pm 0.34$ \\
\hline $\mathrm{C} 20: 3 n 6$ & $1.50 \pm 0.06$ \\
\hline $\mathrm{C} 20: 4 n 6$ & $3.73 \pm 0.20$ \\
\hline $\mathrm{C} 20: 3 n 3$ & $1.71 \pm 0.31$ \\
\hline $\mathrm{C} 20: 5 n 3$ & $1.66 \pm 0.05$ \\
\hline $\mathrm{C} 22: 0$ & $5.89 \pm 0.07$ \\
\hline $\mathrm{C} 22: 1 n 9$ & $3.84 \pm 1.09$ \\
\hline $\mathrm{C} 22: 2 n 6$ & $306.69 \pm 4.63$ \\
\hline $\mathrm{C} 23: 0$ & $20.65 \pm 0.27$ \\
\hline $\mathrm{C} 24: 0$ & $1.82 \pm 1.57$ \\
\hline $\mathrm{C} 24: 1 n 9$ & $1.01 \pm 0.36$ \\
\hline SFA & $1333.33 \pm 0.33$ \\
\hline UFA & $2566.67 \pm 0.33$ \\
\hline MUFA & $1133.89 \pm 1.60$ \\
\hline PUFA & $1432.78 \pm 1.93$ \\
\hline
\end{tabular}

SFA: sum of saturated fatty acids; UFA: sum of unsaturated fatty acids; PUFA: sum of polyunsaturated fatty acids; MUFA: sum of monounsaturated fatty acids.

the fatty acids were: initial oven temperature: $50{ }^{\circ} \mathrm{C}$ for 1 minute; $\operatorname{ramp~} 1: 5^{\circ} \mathrm{C} \cdot \mathrm{min}^{-1}$ to $248^{\circ} \mathrm{C}$; $\operatorname{ramp} 2$ : $248^{\circ} \mathrm{C}$ for 6 minutes.
The gasses used were air $\left(350 \mathrm{~mL} \cdot \mathrm{min}^{-1}\right)$, hydrogen $\left(335 \mathrm{~mL} \cdot \mathrm{min}^{-1}\right)$ and helium $\left(30 \mathrm{~mL} \cdot \mathrm{min}^{-1}\right)$.

Each fatty acid was expressed as a percentage of the total fatty acids. The indices for the activities of $\Delta 9, \Delta 6$ and $\Delta 5$ desaturase and the elongase activity were determined as described by Ntawubizi et al. (2009). The thioesterase index was calculated according to Zhang et al. (2007).

\subsection{Cholesterol and retinol analyses}

Tissue samples and commercial compound feed were saponified according to the procedure described by Saldanha et al. (2006), the unsaponifiable material was extracted by the procedure of López-Cervantes et al. (2006) and cholesterol and retinol contents were quantified by high performance liquid chromatography (HPLC), using a Thermo Finnigan HPLC (Thermo Finnigan, Rodano, Italy) equipped with an on-line solvent degasser (model Spectrasystem SCM1000), solvent delivery system (model Spectrasystem P4000), autosampler with a column oven (model Spectrasystem AS3000), rheodyne injector with a $100 \mu \mathrm{L}$ loop, photodiode array detector (model Spectrasystem UV6000LP) and a system controller ChromQuest 4.1. The analytical column was Ultrasphere ODS $(250 \mathrm{~mm} \times 4.6 \mathrm{~mm}$ I.D.) with a particle size $5 \mu \mathrm{m}$ (Beckman, Fullerton, USA).

The HPLC conditions were as follows: mobile phase 68:28:4 (v/v/v) methanol:acetonitrile:water; a flow rate of $2 \mathrm{~mL} \cdot \mathrm{min}^{-1}$; column temperature $36{ }^{\circ} \mathrm{C}$. The detection was operated using two channels of a diode-array spectrophotometer, $325 \mathrm{~nm}$ for retinol and $208 \mathrm{~nm}$ for cholesterol. Retinol and cholesterol in the non-saponifiable fractions of the lipid fraction were identified by the comparison of retention times and UV absorption spectra with those obtained for corresponding standards (Sigma Chemical Co., Saint Louis, MO, USA). Results are expressed as $\mathrm{mg} \cdot 100 \mathrm{~g}^{-1}$ of sample for cholesterol and $\mu \mathrm{g} \cdot \mathrm{g}^{-1}$ of sample for retinol.

All samples and patterns of fatty acids, cholesterol and retinol were injected at least in duplicate. Repeatability tests were performed injecting a pattern and a sample consecutively six times in a day. Reproducibility tests were also carried out, injecting the pattern and the sample twice a day for 3 days, under the same experimental conditions. Significant differences were not found among the results obtained in any of the tests.

\subsection{Statistical analysis}

Mean and standard error of the mean values were determined for all the parameters and the results were expressed as mean and standard error of the mean for six samples in each group. Analysis of variance (ANOVA) was carried out in order to 
compare the value of each parameter and the significance is given as $\mathrm{P}<0.05, \mathrm{P}<0.01$ and $\mathrm{P}<0.001$. Means were compared by the least-square difference test (LSD), using the computer programme Statistica ${ }^{\odot} 7$ for Windows (Statsoft Inc., Tulsa, OK, USA).

\section{RESULTS}

Table 2 shows the fatty acid composition of total, neutral and polar lipids, cholesterol and intramuscular fat content from the Longissimus dorsi-LD- and Psoas major-PM- muscles.

The intramuscular fat of Celta pigs had a high concentration of saturated (SFA) and monounsaturated (MUFA) fatty acids and a lower concentration of polyunsaturated (PUFA) fatty acids. Oleic acid (C18:1 c n-9) was the most abundant fatty acid in the total and neutral lipids and linoleic acid (C18:2 $n-6)$ in the polar lipids. In polar lipids, the arachidonic acid (C20:4 n-6) content was higher than in total and neutral lipids.

A statistical analysis of the results showed that the total amount of SFA, unsaturated fatty acids (UFA), MUFA and PUFA in the intramuscular fat was affected by the type of muscle. LD showed higher levels of UFA and MUFA and lower levels of SFA and PUFA than the PM intramuscular fat. These differences were a result of the effect on individual fatty acids. For example, palmitoleic (C16:1 n-7), margaric (C17:0), C18:1 c $n-9, \mathrm{C} 18: 2$ $n$-6, $\alpha$-linolenic (C18:3 n-3), arachidic (C20:0), eicosatrienoic (C20:3 n-6), C20:4 n-6, behenic (C22:0) or lignoceric $(\mathrm{C} 24: 0)$ acids showed significant differences $(\mathrm{P}<0.001)$ in the total lipids of the intramuscular fat.

The effects of the type of muscle on the polar lipids from the intramuscular fat were minor. The highest differences between LD and PM muscles were observed for stearic acid (C18:0) and C18:1 c $n$-9.

Fatty acid contents were influenced more by type of muscle than by sex. In the case of total lipid content in the intramuscular fat, only C17:0 showed highly significant $(\mathrm{P}<0.001)$ sex effects.

The neutral lipids of the intramuscular fat showed significant differences for pentadecanoic acid (C15:0), cis-10 heptadecanoic acid (C17:1), C17:0, C18:1 c n-9, C18:3n-3 and cis- 11, 14, 17 eicosatrienoic acid (C20:3 n-3) among males and females.

The effect of sex on the polar lipids of the intramuscular fat was minor, and there were significant differences between male and female animals only concerning the nervonic acid (C24:1 n-9). An interaction between type of muscle and sex was observed for $\mathrm{C} 18: 0$ in the polar lipids.

The fatty acid composition of total lipids, cholesterol and retinol contents from the subcutaneous fat (rump, dorsal, ventral and covering the Biceps femoris muscle) are shown in Table 3 and the fatty acid composition of neutral and polar lipids, are shown in Tables 4 and 5, respectively.

Significant differences were also observed among the fatty acid composition of the subcutaneous fat locations. The fatty acids of total and neutral lipids were affected more by location than polar lipids. The subcutaneous ventral fat showed a higher SFA content and lower UFA, MUFA and PUFA than the subcutaneous dorsal fat, subcutaneous fat from the rump and the fat covering the $B$. femoris muscle. In addition, the polar lipids of the subcutaneous fat showed higher levels of MUFA than in other locations.

Sex affected the fatty acids of the subcutaneous fat. However, in total lipids, there were significant differences for lauric acid (C12:0), C15:0, C17:0, C17:1 and C18:3 n-6 among males and females. In the neutral lipids of the subcutaneous fat, males had a higher content of $\mathrm{C} 12: 0$, myristoleic acid (C14:1), palmitic acid (C16:0), C17:0, C17:1 and cis-13, 16-docosadienoic acid (C22:2 n-6) than females.

An interaction between location and sex was observed for SFA, UFA and MUFA in polar lipids. In polar lipids, the males showed higher contents of C16:1 n-7, C17:1, C18:3n-3 and C20:3 n-3 than the females.

The fatty acid composition of total, neutral and polar lipids, cholesterol and retinol contents from perirenal and hepatic fat are presented in Table 6

In total and neutral lipids, the hepatic fat showed higher levels of PUFA and lower levels of MUFA than the subcutaneous or intramuscular fats. These differences are a direct consequence of higher amounts of some fatty acids such as C18:2 n-6 or C20:4 n-6 in the hepatic fat and lower amounts of $\mathrm{C} 18: 1 \mathrm{c} n-9$, vaccenic acid (C18:1 c $n$-7) or cis-11-eicosenoic acid (C20:1 n-9). In polar lipids, hepatic fat showed higher levels of PUFA than the subcutaneous fat but lower than the intramuscular fat.

The sex had no significant influence on the composition of the hepatic fat.

The SFA (mainly C16:0 and C18:0) and C18:3 $n-3$ contents were higher in the perirenal fat compared to the other fat depots.

Desaturase and elongase activity indices are given in Table 7. $\Delta 6$ desaturase index and C18:0/C16:0 elongase index were not significantly influenced by the location or the sex in LD and PM. However, significant differences $(\mathrm{P}<0.01)$ were observed between males and females in the rump.

A significant effect $(\mathrm{P}<0.001)$ of location and sex was observed for the $\Delta 5$ desaturase index in LD and PM. PM presented a higher $\Delta 5$ desaturase index than LD in females than in males. In subcutaneous fat, differences were determined only by the location in the $\Delta 5$ desaturase index. 


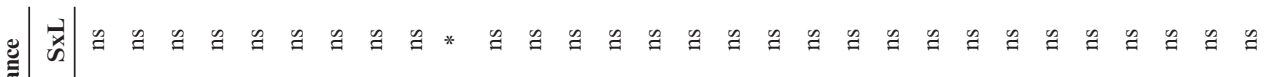

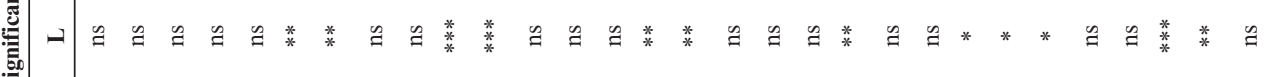

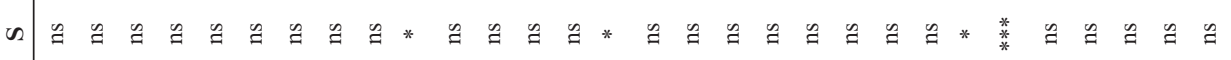

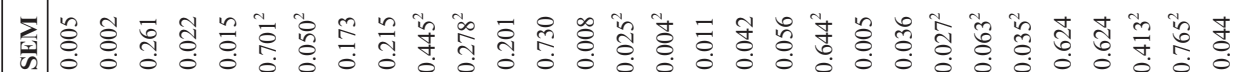

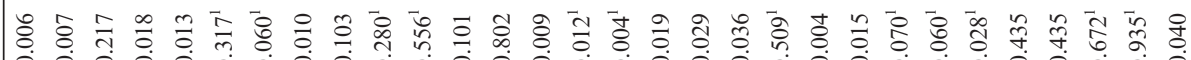

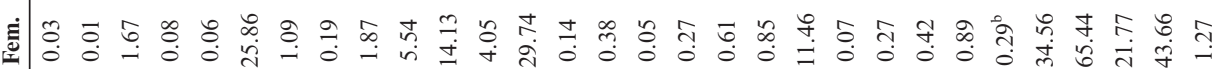

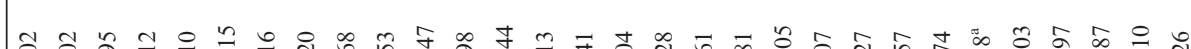
श्ञ

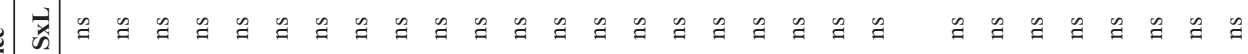

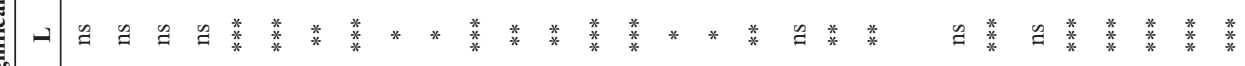

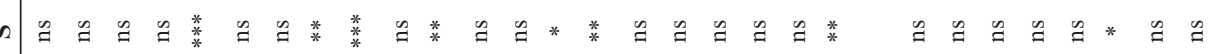

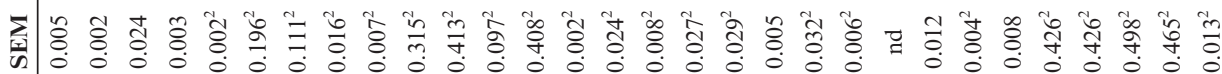
掊

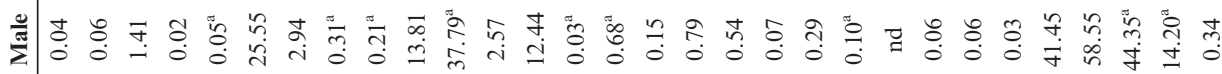

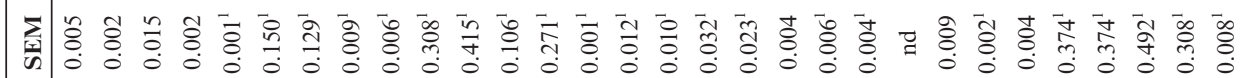

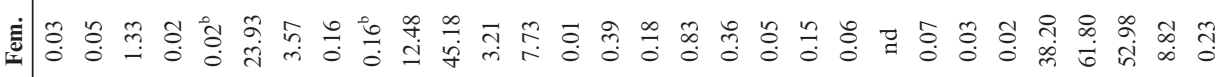

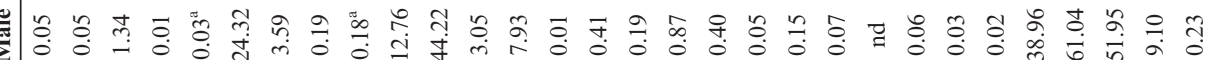

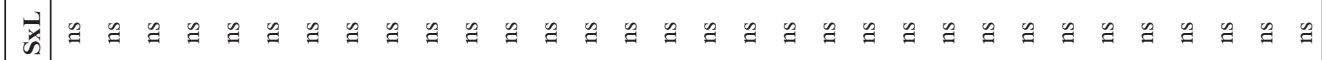

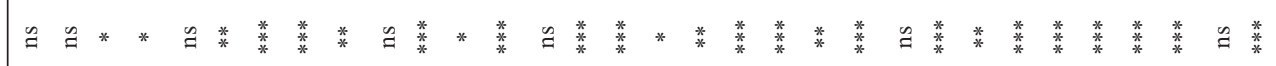

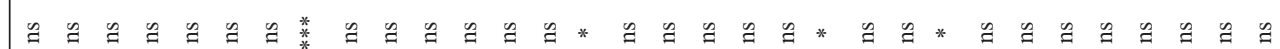

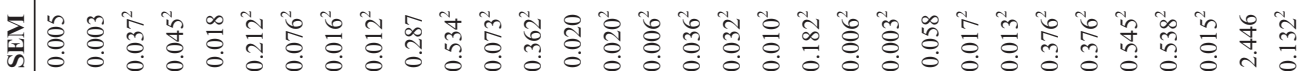

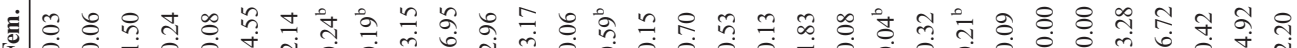

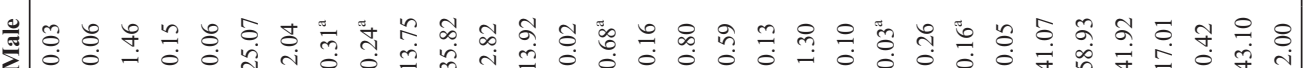

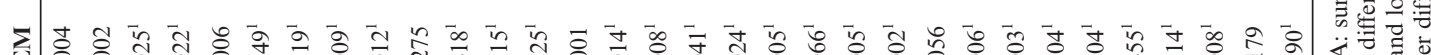

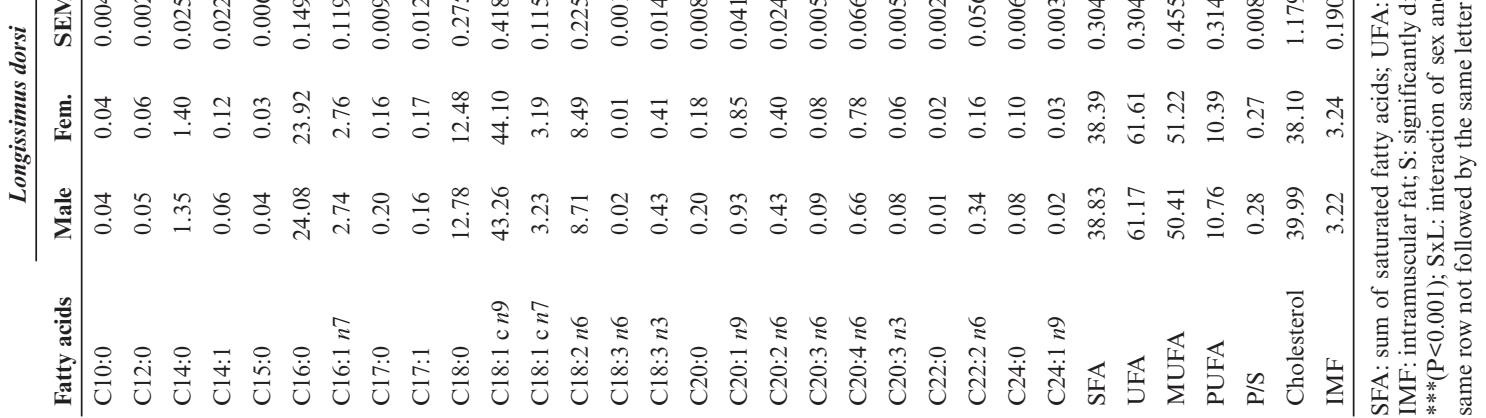


TABLE 3. Fatty acid composition (as a percentage of total fatty acids), cholesterol ( $\mathrm{mg} \cdot 100 \mathrm{~g}^{-1}$ of sample), retinol ( $\mu \mathrm{g} \cdot \mathrm{g}^{-1} \mathrm{of}$ sample) and standard error of the mean (SEM) of total lipids from subcutaneous fat

\begin{tabular}{|c|c|c|c|c|c|c|c|c|c|c|c|c|c|c|c|}
\hline \multirow[b]{2}{*}{ Fatty acids } & \multicolumn{3}{|c|}{ Rump } & \multicolumn{3}{|c|}{ Sub. Biceps femoris } & \multicolumn{3}{|c|}{ Subcutaneous dorsal } & \multicolumn{3}{|c|}{ Subcutaneous ventral } & \multicolumn{3}{|c|}{ Significance } \\
\hline & Male & Fem. & SEM & Male & Fem. & SEM & Male & Fem. & SEM & Male & Fem. & SEM & $\mathbf{S}$ & $\mathbf{L}$ & SxL \\
\hline C10:0 & 0.01 & 0.02 & 0.002 & 0.01 & 0.01 & 0.002 & 0.01 & 0.01 & 0.001 & 0.01 & 0.01 & 0.002 & $\mathrm{~ns}$ & $\mathrm{~ns}$ & $\mathrm{~ns}$ \\
\hline $\mathrm{C} 12: 0$ & 0.04 & 0.04 & $0.002^{1}$ & 0.04 & 0.04 & $0.001^{1}$ & 0.04 & 0.03 & $0.001^{1}$ & $0.06^{\mathrm{a}}$ & $0.05^{\mathrm{b}}$ & $0.003^{2}$ & $* *$ & $* * *$ & $\mathrm{~ns}$ \\
\hline $\mathrm{C} 14: 0$ & 1.19 & 1.12 & $0.028^{1}$ & 1.16 & 1.11 & $0.024^{1}$ & 1.11 & 1.05 & $0.020^{1}$ & 1.32 & 1.25 & $0.035^{2}$ & $*$ & $* * *$ & ns \\
\hline $\mathrm{C} 14: 1$ & 0.01 & 0.01 & 0.001 & 0.01 & 0.01 & 0.001 & 0.01 & 0.01 & 0.001 & 0.01 & 0.01 & 0.001 & $*$ & $* *$ & ns \\
\hline C15:0 & $0.04^{\mathrm{a}}$ & $0.03^{\mathrm{b}}$ & $0.001^{1}$ & 0.03 & 0.03 & $0.001^{1}$ & $0.04^{\mathrm{a}}$ & $0.04^{\mathrm{b}}$ & $0.002^{2}$ & $0.05^{\mathrm{a}}$ & $0.04^{\mathrm{b}}$ & $0.002^{2}$ & $* * *$ & $* * *$ & ns \\
\hline C16:0 & 22.25 & 21.79 & $0.296^{1}$ & 21.93 & 21.44 & $0.187^{1}$ & 22.58 & 21.80 & $0.273^{1}$ & 25.27 & 24.44 & $0.401^{2}$ & $*$ & $* * *$ & $\mathrm{~ns}$ \\
\hline $\mathrm{C} 16: 1 n 7$ & 1.72 & 1.60 & $0.054^{1}$ & 2.09 & 2.09 & $0.145^{2}$ & 1.73 & 1.57 & $0.059^{1}$ & 2.12 & 2.20 & $0.107^{2}$ & ns & $* * *$ & $\mathrm{~ns}$ \\
\hline $\mathrm{C} 17: 0$ & 0.26 & 0.23 & $0.009^{12}$ & 0.23 & 0.22 & $0.009^{2}$ & 0.30 & 0.26 & $0.013^{1}$ & $0.31^{\mathrm{a}}$ & $0.24^{\mathrm{b}}$ & $0.016^{1}$ & $* *$ & $* *$ & $\mathrm{~ns}$ \\
\hline $\mathrm{C} 17: 1$ & $0.26^{\mathrm{a}}$ & $0.21^{\mathrm{b}}$ & 0.013 & 0.28 & 0.25 & 0.010 & 0.27 & 0.22 & 0.018 & 0.26 & 0.23 & 0.010 & $* *$ & $\mathrm{~ns}$ & $\mathrm{~ns}$ \\
\hline C18:0 & 11.92 & 11.92 & $0.199^{1}$ & 11.45 & 11.34 & $0.256^{1}$ & 11.89 & 12.24 & $0.312^{1}$ & 13.92 & 12.67 & $0.486^{2}$ & ns & $* *$ & $\mathrm{~ns}$ \\
\hline $\mathrm{C} 18: 1 c n 9$ & 43.98 & 44.68 & $0.479^{1}$ & 46.36 & 46.29 & $0.393^{2}$ & 43.02 & 43.71 & $0.546^{1}$ & 39.45 & 41.76 & $0.817^{3}$ & $\mathrm{~ns}$ & $* * *$ & $\mathrm{~ns}$ \\
\hline $\mathrm{C} 18: 1 c n 7$ & 2.13 & 2.19 & $0.077^{1}$ & 2.48 & 2.45 & $0.098^{2}$ & 2.15 & 2.16 & $0.058^{1}$ & 2.20 & 2.45 & $0.107^{12}$ & $\mathrm{~ns}$ & $\mathrm{~ns}$ & $\mathrm{~ns}$ \\
\hline $\mathrm{C} 18: 2 n 6$ & 12.32 & 12.43 & $0.119^{1}$ & 10.74 & 11.49 & $0.235^{2}$ & 13.16 & 13.06 & $0.281^{1}$ & 11.79 & 11.26 & $0.351^{2}$ & $\mathrm{~ns}$ & $* *$ & $\mathrm{~ns}$ \\
\hline $\mathrm{C} 18: 3$ n6 & $0.02^{\mathrm{a}}$ & $0.01^{\mathrm{b}}$ & 0.002 & 0.02 & 0.02 & 0.001 & $0.03^{\mathrm{a}}$ & $0.02^{\mathrm{b}}$ & 0.002 & 0.02 & 0.02 & & $* *$ & $\mathrm{~ns}$ & ns \\
\hline $\mathrm{C} 18: 3$ & 0.64 & 0.59 & & 0.61 & & 0.017 & 0.66 & 0.64 & 0.028 & 0.61 & 0.56 & 0.024 & $\mathrm{~ns}$ & $\mathrm{~ns}$ & ns \\
\hline $\mathrm{C} 20: 0$ & 0.22 & 0.20 & $0.010^{12}$ & 0.17 & 0.15 & $0.008^{2}$ & 0.23 & 0.21 & $0.021^{1}$ & 0.21 & 0.17 & $0.013^{12}$ & ns & $*$ & $\mathrm{~ns}$ \\
\hline $\mathrm{C} 20: 1 n 9$ & 1.31 & 1.36 & $0.040^{1}$ & 1.06 & 1.09 & $0.020^{2}$ & 1.25 & 1.35 & $0.071^{1}$ & 1.02 & 0.99 & $0.045^{2}$ & $\mathrm{~ns}$ & $* * *$ & ns \\
\hline $\mathrm{C} 20: 2 n 6$ & 0.85 & 0.85 & $0.025^{1}$ & 0.62 & 0.65 & $0.018^{2}$ & 0.83 & 0.81 & $0.030^{1}$ & 0.56 & 0.54 & $0.037^{3}$ & $\mathrm{~ns}$ & $* * *$ & $\mathrm{~ns}$ \\
\hline $\mathrm{C} 20: 3$ n6 & 0.07 & 0.06 & 0.004 & 0.07 & 0.07 & 0.004 & 0.07 & 0.07 & 0.004 & 0.07 & 0.07 & 0.005 & $\mathrm{~ns}$ & $\mathrm{~ns}$ & $\mathrm{~ns}$ \\
\hline $\mathrm{C} 20: 4$ n6 & 0.12 & 0.11 & $0.006^{1}$ & 0.16 & 0.16 & $0.008^{2}$ & 0.12 & 0.12 & $0.008^{1}$ & 0.19 & 0.18 & $0.011^{3}$ & $\mathrm{~ns}$ & $* * *$ & ns \\
\hline $\mathrm{C} 20: 3 n 3$ & 0.18 & 0.16 & $0.009^{1}$ & 0.12 & 0.11 & $0.004^{2}$ & 0.15 & 0.15 & $0.006^{3}$ & 0.10 & 0.09 & $0.006^{2}$ & $\mathrm{~ns}$ & $* * *$ & $\mathrm{~ns}$ \\
\hline $\mathrm{C} 22: 2 n 6$ & 0.43 & 0.36 & 0.062 & 0.31 & 0.30 & 0.054 & 0.31 & 0.45 & 0.058 & 0.38 & 0.39 & 0.066 & $\mathrm{~ns}$ & $\mathrm{~ns}$ & $\mathrm{~ns}$ \\
\hline C24:0 & 0.03 & 0.03 & $0.003^{1}$ & 0.05 & 0.04 & $0.004^{12}$ & 0.04 & 0.03 & $0.003^{1}$ & 0.05 & 0.05 & $0.004^{2}$ & ns & $* *$ & $\mathrm{~ns}$ \\
\hline $\mathrm{C} 24: 1 n 9$ & nd & 0.01 & 0.002 & 0.01 & 0.01 & 0.002 & nd & 0.01 & 0.002 & 0.01 & 0.01 & 0.001 & $*$ & $\mathrm{~ns}$ & ns \\
\hline SFA & 35.96 & 35.38 & $0.436^{1}$ & 35.07 & 34.38 & $0.276^{1}$ & 36.24 & 35.68 & $0.494^{1}$ & $41.19^{\mathrm{a}}$ & $38.92^{\mathrm{b}}$ & $0.808^{2}$ & $\mathrm{~ns}$ & $* * *$ & ns \\
\hline UFA & 64.04 & 64.62 & $0.436^{1}$ & 64.93 & 65.62 & $0.276^{1}$ & 63.76 & 64.32 & $0.494^{1}$ & 58.81 & 60.75 & $0.753^{2}$ & $\mathrm{~ns}$ & $* * *$ & $\mathrm{~ns}$ \\
\hline MUFA & 49.41 & 50.06 & $0.410^{1}$ & 52.29 & 52.19 & $0.400^{2}$ & 48.43 & 49.02 & $0.585^{1}$ & $45.08^{\mathrm{a}}$ & $47.64^{\mathrm{b}}$ & $0.918^{3}$ & $\mathrm{~ns}$ & $* * *$ & ns \\
\hline PUFA & 14.63 & 14.57 & $0.127^{1}$ & 12.64 & 13.43 & $0.271^{2}$ & 15.33 & 15.30 & $0.325^{1}$ & 13.73 & 13.11 & $0.433^{2}$ & $\mathrm{~ns}$ & $* * *$ & $\mathrm{~ns}$ \\
\hline $\mathrm{P} / \mathrm{S}$ & 0.41 & 0.41 & $0.007^{1}$ & 0.36 & 0.39 & $0.008^{2}$ & 0.42 & 0.43 & $0.011^{1}$ & 0.33 & 0.34 & $0.011^{3}$ & ns & $* * *$ & $\mathrm{~ns}$ \\
\hline Cholesterol & 68.72 & 73.74 & 5.120 & 71.33 & 61.30 & 4.307 & 82.77 & 74.61 & 7.144 & 85.27 & 85.27 & 3.470 & $\mathrm{~ns}$ & $\mathrm{~ns}$ & ns \\
\hline Retinol & 1.41 & 1.67 & 0.107 & 1.07 & 1.12 & 0.065 & 1.57 & 1.67 & 0.142 & 1.25 & 1.44 & 0.100 & $\mathrm{~ns}$ & $\mathrm{~ns}$ & $\mathrm{~ns}$ \\
\hline
\end{tabular}

SFA: sum of saturated fatty acids; UFA: sum of unsaturated fatty acids; PUFA: sum of polyunsaturated fatty acids; MUFA: sum of monounsaturated fatty acids; P/S: the ratio of total PUFA to total SFA; Fem: female; S: significantly different values as influenced by sex $*(\mathrm{P}<0.05) ; * *(\mathrm{P}<0.01) ; * * *(\mathrm{P}<0.001)$; ns: no significant difference; L: significantly different values as influenced by location $*(\mathrm{P}<0.05) ;{ }^{* *}(\mathrm{P}<0.01) ; * *(\mathrm{P}<0.001)$; SxL: interaction of sex and location $*(\mathrm{P}<0.05) ; * *(\mathrm{P}<0.01) ; * *(\mathrm{P}<0.001) ;{ }^{\mathrm{a}-\mathrm{b}}$ Means within the same row not followed by the same letter differ significantly (influence of sex $(\mathrm{P}<0.05) ;{ }^{1-3} \mathrm{SEM}$ within the same row not followed by the same letter differ significantly (influence of location) $(\mathrm{P}<0.05)$.

The $\Delta 9$ desaturase index was higher in LD than in PM, showing that the conversion of palmitic acid to palmitoleic acid and stearic acid to oleic acid is higher in LD. This index was also higher in fat covering the Biceps femoris muscle than in other locations of subcutaneous fat. This index did not differ between the two sexes.

Elongase activity did not show differences between females and males in LD and PM. However, the elongase index, which provided an estimate of C16:1 n-7 to C18:1 c n-7 fatty acid elongase activity, was higher in PM than in LD. In the subcutaneous fat, significant differences were observed between sex $(\mathrm{P}<0.05)$ and locations $(\mathrm{P}<0.001)$. Finally, the thioesterase index did not differ among locations or sexes in intramuscular fat. In this case, differences between locations were observed concerning subcutaneous fat. 
TABLE 4. Fatty acid composition (as a percentage of total fatty acids) and standard error of the mean (SEM) of neutral lipids from subcutaneous fat

\begin{tabular}{|c|c|c|c|c|c|c|c|c|c|c|c|c|c|c|c|}
\hline \multirow[b]{2}{*}{ Fatty acids } & \multicolumn{3}{|c|}{ Rump } & \multicolumn{3}{|c|}{ Sub. Biceps femoris } & \multicolumn{3}{|c|}{ Subcutaneous dorsal } & \multicolumn{3}{|c|}{ Subcutaneous ventral } & \multicolumn{3}{|c|}{ Significance } \\
\hline & Male & Fem. & SEM & Male & Fem. & SEM & Male & Fem. & SEM & Male & Fem. & SEM & $\mathbf{S}$ & $\mathbf{L}$ & SxL \\
\hline $\mathrm{C} 10: 0$ & 0.01 & 0.02 & 0.002 & 0.01 & 0.02 & 03 & 0.01 & 0.02 & 0.002 & 0.01 & 0.02 & 0.002 & $*$ & ns & ns \\
\hline $\mathrm{C} 12: 0$ & 0.05 & 0.04 & $0.002^{1}$ & 0.05 & 0.04 & $0.002^{1}$ & 0.04 & 0.04 & $0.001^{1}$ & 0.06 & 0.05 & $0.001^{2}$ & $*$ & $* * *$ & ns \\
\hline $\mathrm{C} 14: 0$ & 1.24 & 1.18 & $0.027^{1}$ & 1.22 & 1.18 & $0.021^{1}$ & 1.18 & 1.12 & $0.020^{1}$ & 1.36 & 1.37 & $0.020^{2}$ & ns & $* * *$ & ns \\
\hline $\mathrm{C} 14: 1$ & 0.02 & 0.01 & 0.003 & 0.02 & 0.01 & 0.003 & 0.01 & 0.01 & 003 & 0.02 & 0.01 & 0.003 & $*$ & ns & ns \\
\hline $\mathrm{C} 15: 0$ & 0.04 & 0.05 & 0.008 & 0.03 & 0.03 & 0.001 & $0.04^{\mathrm{a}}$ & $0.03^{\mathrm{b}}$ & 0.002 & $0.04^{\mathrm{a}}$ & $0.03^{\mathrm{b}}$ & 0.002 & $\mathrm{~ns}$ & ns & ns \\
\hline C16:0 & 22.32 & 21.85 & $0.281^{1}$ & 22.15 & 21.82 & $0.160^{1}$ & 22.75 & 21.91 & $0.312^{1}$ & $25.34^{\mathrm{a}}$ & $24.15^{\mathrm{b}}$ & $0.312^{2}$ & $*$ & $* * *$ & ns \\
\hline $\mathrm{C} 16: 1 n 7$ & 2.40 & 2.18 & $0.086^{1}$ & 2.81 & 2.68 & $0.096^{2}$ & 2.43 & 2.24 & $0.062^{1}$ & 2.90 & 3.03 & $0.062^{2}$ & ns & $* * *$ & ns \\
\hline $\mathrm{C} 17: 0$ & 0.26 & 0.23 & $0.009^{12}$ & 0.23 & 0.22 & $0.008^{1}$ & 0.29 & 0.26 & $0.013^{2}$ & $0.30^{\mathrm{a}}$ & $0.24^{\mathrm{b}}$ & $0.013^{2}$ & $* *$ & $* *$ & ns \\
\hline $\mathrm{C} 17: 1$ & $0.25^{\mathrm{a}}$ & $0.20^{\mathrm{b}}$ & 0.013 & 0.26 & 0.23 & 0.010 & 0.25 & 0.21 & 0.018 & 0.24 & 0.21 & 0.018 & $* *$ & ns & ns \\
\hline C18:0 & 12.36 & 13.22 & $0.372^{1}$ & 10.90 & 10.71 & $0.261^{2}$ & 11.83 & 11.74 & $0.269^{3}$ & $13.33^{\mathrm{a}}$ & $11.87^{\mathrm{b}}$ & $0.269^{13}$ & ns & $* * *$ & ns \\
\hline $\mathrm{C} 18: 1 c n 9$ & 43.13 & 43.20 & $0.508^{1}$ & 46.23 & 46.29 & $0.417^{2}$ & 42.49 & 43.87 & $0.488^{1}$ & $39.59^{\mathrm{a}}$ & $42.70^{\mathrm{b}}$ & $0.488^{3}$ & $\mathrm{~ns}$ & $* * *$ & ns \\
\hline $\mathrm{C} 18: 1 c n 7$ & 2.06 & 2.03 & $0.086^{1}$ & 2.38 & 2.42 & $0.105^{2}$ & 1.96 & 1.98 & $0.108^{1}$ & 2.28 & 2.41 & $0.108^{2}$ & $\mathrm{~ns}$ & $* *$ & ns \\
\hline $\mathrm{C} 18: 2$ n6 & 12.49 & 12.44 & $0.162^{1}$ & 10.93 & 11.56 & $0.229^{2}$ & 13.34 & 13.24 & $0.293^{3}$ & 11.78 & 11.30 & $0.293^{2}$ & ns & $* * *$ & ns \\
\hline $\mathrm{C} 18: 3 n 6$ & 0.01 & 0.02 & 0.005 & 0.01 & 0.01 & 0.002 & 0.02 & 0.02 & 0.006 & 0.02 & 0.02 & 0.006 & ns & ns & ns \\
\hline $\mathrm{C} 18: 3 n 3$ & 0.65 & 0.60 & $0.020^{12}$ & 0.62 & 0.62 & $0.013^{12}$ & 0.68 & 0.64 & $0.028^{1}$ & 0.62 & 0.57 & $0.028^{2}$ & $\mathrm{~ns}$ & ns & ns \\
\hline $\mathrm{C} 20: 0$ & 0.19 & 0.20 & $0.009^{13}$ & 0.15 & 0.13 & $0.007^{23}$ & 0.22 & 0.20 & $0.019^{1}$ & 0.18 & 0.16 & $.019^{3}$ & $\mathrm{~ns}$ & $* *$ & $\mathrm{~ns}$ \\
\hline $\mathrm{C} 20: 1 n 9$ & 1.21 & 1.31 & $0.040^{1}$ & 0.96 & 0.98 & $0.024^{2}$ & 1.18 & 1.28 & $0.071^{1}$ & 0.89 & 0.95 & $0.071^{2}$ & ns & $* * *$ & ns \\
\hline $\mathrm{C} 20: 2 n 6$ & 0.82 & 0.81 & $0.022^{1}$ & 0.60 & 0.60 & $0.013^{2}$ & 0.80 & 0.81 & $0.015^{1}$ & 0.54 & 0.51 & $0.015^{3}$ & $\mathrm{~ns}$ & $* * *$ & ns \\
\hline $\mathrm{C} 20: 3 n 6$ & 0.06 & 0.06 & 0.006 & 0.06 & 0.07 & 0.004 & 0.06 & 0.0 & 0.005 & 0.06 & 0.06 & 0.005 & $\mathrm{~ns}$ & ns & ns \\
\hline $\mathrm{C} 20: 4$ n6 & 0.11 & 0.11 & $0.009^{1}$ & 0.15 & 0.14 & $0.008^{2}$ & 0.11 & 0.10 & $0.007^{1}$ & 0.17 & 0.16 & $0.007^{2}$ & ns & $* * *$ & ns \\
\hline $\mathrm{C} 20: 3 n 3$ & 0.17 & 0.15 & $0.008^{1}$ & 0.10 & 0.10 & $0.004^{2}$ & 0.12 & 0.14 & $0.013^{3}$ & 0.09 & 0.08 & $0.013^{3}$ & $\mathrm{~ns}$ & $* * *$ & ns \\
\hline $\mathrm{C} 22: 2$ n6 & 0.10 & 0.07 & 0.016 & 0.09 & 0.06 & 0.013 & $0.14^{\mathrm{a}}$ & $0.05^{\mathrm{b}}$ & 0.032 & $0.15^{\mathrm{a}}$ & $0.06^{\mathrm{b}}$ & 0.032 & $* *$ & $\mathrm{~ns}$ & $\mathrm{~ns}$ \\
\hline $\mathrm{C} 24: 0$ & 0.03 & 0.03 & $0.001^{1}$ & 0.03 & 0.04 & $0.003^{2}$ & 0.03 & 0.03 & $0.003^{12}$ & 0.04 & 0.04 & $0.003^{2}$ & $\mathrm{~ns}$ & $*$ & ns \\
\hline $\mathrm{C} 24: 1 n 9$ & 0.01 & 0.01 & 0.004 & 0.02 & 0.01 & 0.004 & 0.01 & 0.01 & 0.002 & 0.01 & 0.02 & 0.002 & $\mathrm{~ns}$ & ns & ns \\
\hline SFA & 36.51 & 36.81 & $0.454^{1}$ & 34.77 & 34.19 & $0.346^{2}$ & 36.40 & 35.34 & $0.507^{12}$ & $40.66^{\mathrm{a}}$ & $37.94^{\mathrm{b}}$ & $0.507^{3}$ & ns & $* * *$ & ns \\
\hline UFA & 63.49 & 63.19 & $0.454^{1}$ & 65.23 & 65.81 & $0.346^{2}$ & 63.60 & 64.66 & $0.507^{12}$ & $59.34^{\mathrm{a}}$ & $62.06^{\mathrm{b}}$ & $0.507^{3}$ & ns & $* * *$ & ns \\
\hline MUFA & 49.08 & 48.94 & $0.465^{1}$ & 52.67 & 52.64 & $0.428^{2}$ & 48.33 & 49.60 & $0.530^{1}$ & $45.92^{\mathrm{a}}$ & $49.31^{b}$ & $0.530^{1}$ & $\mathrm{~ns}$ & $* * *$ & ns \\
\hline PUFA & 14.42 & 14.25 & $0.186^{1}$ & 12.55 & 13.17 & $0.249^{2}$ & 15.27 & 15.06 & $0.319^{1}$ & 13.42 & 12.75 & $0.319^{2}$ & $\mathrm{~ns}$ & $* * *$ & ns \\
\hline $\mathrm{P} / \mathrm{S}$ & 0.40 & 0.39 & $0.007^{1}$ & 0.36 & 0.39 & $0.008^{1}$ & 0.42 & 0.43 & $0.012^{2}$ & 0.33 & 0.34 & $0.012^{2}$ & $\mathrm{~ns}$ & $* * *$ & $\mathrm{~ns}$ \\
\hline
\end{tabular}

SFA: sum of saturated fatty acids; UFA: sum of unsaturated fatty acids; PUFA: sum of polyunsaturated fatty acids; MUFA: sum of monounsaturated fatty acids; P/S: the ratio of total PUFA to total SFA; Fem: female; S: significantly different values as influenced by $\operatorname{sex} *(\mathrm{P}<0.05) ; * *(\mathrm{P}<0.01) ; * *(\mathrm{P}<0.001)$; L: significantly different values as influenced by location $*(\mathrm{P}<0.05) ; * *(\mathrm{P}<0.01)$; $* * *(\mathrm{P}<0.001)$; ns: no significant difference; SxL: interaction of sex and location ${ }^{*}(\mathrm{P}<0.05) ; * *(\mathrm{P}<0.01) ; * * *(\mathrm{P}<0.001) ;{ }^{\mathrm{a}-\mathrm{b}} \mathrm{Means}$ within the same row not followed by the same letter differ significantly (influence of sex) $(\mathrm{P}<0.05) ;{ }^{1-3} \mathrm{SEM}$ within the same row not followed by the same letter differ significantly (influence of location) $(\mathrm{P}<0.05)$.

In LD muscle significantly higher values $(\mathrm{P}<0.05)$ of intramuscular fat than in the PM muscle were obsereved (Table 2). In our study, the retinol content in muscles was not detectable.

Cholesterol contents from the subcutaneous and perirenal fat was superior to that described in intramuscular fat. The cholesterol and retinol contents of the subcutaneous locations were not strongly influenced by location. The ventral subcutaneous fat had the greatest $(\mathrm{P}<0.05)$ cholesterol values.
The retinol and cholesterol contents from the hepatic fat were much higher than those observed in other locations.

The percentage of intramuscular fat and cholesterol contents in the muscles and retinol and cholesterol contents in the subcutaneous and perirrenal fat showed no significant differences between males and females. However, there appears to be a greater deposition $(\mathrm{P}<0.05)$ of retinol in the liver of males than in females. 
TABLE 5. Fatty acid composition (as a percentage of total fatty acids) and standard error of the mean (SEM) of polar lipids in subcutaneous fat

\begin{tabular}{|c|c|c|c|c|c|c|c|c|c|c|c|c|c|c|c|}
\hline \multirow[b]{2}{*}{ Fatty acids } & \multicolumn{3}{|c|}{ Rump } & \multicolumn{3}{|c|}{ Sub. Biceps femoris } & \multicolumn{3}{|c|}{ Subcutaneous dorsal } & \multicolumn{3}{|c|}{ Subcutaneous ventral } & \multicolumn{3}{|c|}{ Significance } \\
\hline & Male & Fem. & SEM & Male & Fem. & SEM & Male & Fem. & SEM & Male & Fem. & $\overline{\text { SEM }}$ & $\mathbf{S}$ & $\mathbf{L}$ & SxL \\
\hline C10:0 & 0.04 & 0.04 & 0.008 & 0.05 & 0.03 & 0.010 & 0.05 & 0.07 & 0.016 & 0.06 & 0.05 & 016 & ns & $\mathrm{ns}$ & ns \\
\hline & & & & 0.06 & & & & & & 0.06 & 0.08 & & ns & $\mathrm{ns}$ & ns \\
\hline $\mathrm{C} 14: 0$ & 1.29 & 1.35 & $0.050^{1}$ & 1.29 & 1.31 & $0.038^{1}$ & 1.27 & 1.34 & $0.046^{1}$ & 1.51 & 1.48 & $0.071^{2}$ & ns & $*$ & ns \\
\hline $\mathrm{C} 14: 1$ & 0.02 & 0.03 & 0.006 & 0.02 & 0.02 & .004 & $0.02^{\mathrm{a}}$ & $0.04^{\mathrm{b}}$ & 0.007 & 0.03 & 0.03 & 0.005 & ns & $\mathrm{ns}$ & ns \\
\hline C15:0 & $0.02^{\mathrm{a}}$ & $0.03^{\mathrm{b}}$ & 0.004 & 0.03 & 0.03 & 0.003 & 0.09 & 0.05 & 0.026 & 0.03 & 0.04 & 0.005 & $\mathrm{~ns}$ & $\mathrm{~ns}$ & $\mathrm{~ns}$ \\
\hline $\mathrm{C} 16: 0$ & 23.74 & 24.62 & $0.389^{1}$ & $23.26^{\mathrm{a}}$ & $25.27^{\mathrm{b}}$ & $0.511^{1}$ & 25.27 & 26.87 & $0.566^{2}$ & 27.55 & 25.90 & $0.704^{2}$ & $\mathrm{~ns}$ & $* *$ & ns \\
\hline $\mathrm{C} 16: 1 n 7$ & 1.83 & 1.60 & $0.063^{1}$ & 2.08 & 1.85 & $0.063^{2}$ & $1.77^{\mathrm{a}}$ & $1.51^{\mathrm{b}}$ & $0.062^{1}$ & 1.98 & 2.09 & $0.083^{2}$ & $*$ & $* * *$ & $\mathrm{~ns}$ \\
\hline C17:0 & 0.26 & 0.26 & 0.008 & 0.24 & 0.25 & 0.012 & 0.29 & 0.29 & 0.021 & 0.31 & 0.26 & 0.018 & ns & $\mathrm{ns}$ & ns \\
\hline C17:1 & $0.20^{\mathrm{a}}$ & $0.15^{\mathrm{b}}$ & $0.010^{12}$ & 0.21 & 0.19 & $0.009^{1}$ & $0.19^{\mathrm{a}}$ & $0.14^{\mathrm{b}}$ & $0.015^{2}$ & 0.18 & 0.16 & $0.008^{2}$ & $* * *$ & $*$ & ns \\
\hline C18:0 & 12.93 & 13.90 & $0.304^{1}$ & 12.29 & 12.66 & $0.246^{1}$ & 14.40 & 15.33 & $0.662^{2}$ & $15.17^{\mathrm{a}}$ & $13.34^{\mathrm{b}}$ & $0.471^{2}$ & ns & $* *$ & ns \\
\hline $\mathrm{C} 18: 1 c n 9$ & 40.08 & 39.72 & $0.561^{1}$ & 42.65 & 40.52 & $0.606^{1}$ & 37.04 & 35.81 & & $35.81^{\mathrm{a}}$ & $39.11^{\mathrm{b}}$ & & ns & $* * *$ & ns \\
\hline $\mathrm{C} 18: 1 c n 7$ & 2.66 & 2.61 & $0.107^{1}$ & 3.00 & 3.03 & $0.131^{2}$ & 2.54 & 2.61 & $0.138^{1}$ & 2.56 & 3.03 & $9^{12}$ & $\mathrm{~ns}$ & $\mathrm{~ns}$ & ns \\
\hline $\mathrm{C} 18: 2 n 6$ & $12.87^{\mathrm{a}}$ & $12.11^{\mathrm{b}}$ & $0.172^{1}$ & 11.35 & 11.20 & $0.272^{2}$ & 13.00 & 12.16 & $0.349^{1}$ & 11.25 & 11.07 & & $\mathrm{~ns}$ & $* * *$ & ns \\
\hline & & & & & & & & & & & & & $\mathrm{ns}$ & $\mathrm{ns}$ & $\mathrm{ns}$ \\
\hline C18: & 0.61 & 0 . & 0.026 & 0.57 & 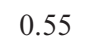 & 0.020 & 0.58 & 0.52 & 0.027 & 0.52 & 0.46 & 0.035 & $*$ & $\mathrm{~ns}$ & ns \\
\hline C20:0 & 0.17 & 0.18 & $0.009^{1}$ & 0.14 & 0.15 & $0.008^{2}$ & 0.19 & 0.20 & $0.009^{1}$ & 0.17 & 0.17 & $0.012^{12}$ & ns & $*$ & ns \\
\hline $\mathrm{C} 20: 1 n 9$ & 1.07 & 1.01 & $0.033^{1}$ & 0.92 & 0.88 & $0.022^{2}$ & 1.01 & 0.99 & $0.040^{1}$ & 0.86 & 0.81 & $0.045^{2}$ & ns & $* * *$ & ns \\
\hline & 0.75 & 0.72 & $0.020^{1}$ & 0.58 & 0.5 & $017^{2}$ & 0.6 & & $0.030^{3}$ & 0.51 & 0.49 & & ns & $* * *$ & ns \\
\hline :3 n6 & 0.12 & 0.18 & 0.019 & 0.11 & 0.15 & 0.012 & 0.15 & 0.20 & 0.029 & 0.16 & 0.15 & 0.022 & ns & $\mathrm{ns}$ & ns \\
\hline $\mathrm{C} 20: 4$ n6 & 0.39 & 0.34 & 0.050 & 0.49 & 0.32 & 0.067 & 0.49 & 0.34 & 0.071 & 0.39 & 0.48 & 1 & $\mathrm{~ns}$ & $\mathrm{~ns}$ & $\mathrm{~ns}$ \\
\hline $\mathrm{C} 20: 3 n 3$ & 0.14 & 0.10 & $0.011^{1}$ & 0.09 & 0.07 & $0.007^{2}$ & 0.10 & 0.09 & $0.013^{12}$ & 0.10 & 0.07 & $0.014^{2}$ & $*$ & $\mathrm{~ns}$ & ns \\
\hline $\mathrm{C} 22: 2$ n6 & 0.65 & 0.33 & 0.085 & 0.44 & 0.70 & 0.108 & 0.69 & 0.56 & 0.091 & 0.66 & 0.59 & 0.085 & ns & $\mathrm{ns}$ & ns \\
\hline $\mathrm{C} 24: 0$ & 0.06 & 0.07 & 0.009 & 0.07 & 0.11 & 0.020 & 0.08 & 0.09 & 0.010 & 0.09 & 0.09 & 0.007 & ns & $\mathrm{ns}$ & ns \\
\hline $\mathrm{C} 24: 1 n 9$ & 0.03 & 0.04 & 0.010 & 0.03 & 0.06 & 0.018 & 0.04 & 0.04 & 0.008 & 0.03 & 0.04 & 0.008 & ns & $\mathrm{ns}$ & $\mathrm{ns}$ \\
\hline SFA & 38.55 & 40.49 & $0.641^{1}$ & 37.43 & 39.87 & $0.698^{1}$ & 41.69 & 44.30 & $1.100^{2}$ & $44.94^{\mathrm{a}}$ & $41.41^{\mathrm{b}}$ & $1.135^{2}$ & $\mathrm{~ns}$ & $* * *$ & $*$ \\
\hline UFA & 61.45 & 59.51 & $0.641^{1}$ & 62.57 & 60.13 & $0.698^{1}$ & 58.31 & 55.70 & $1.100^{2}$ & $55.06^{\mathrm{a}}$ & $58.59^{\mathrm{b}}$ & $1.135^{2}$ & $\mathrm{~ns}$ & $* * *$ & $*$ \\
\hline MUFA & 45.89 & 45.18 & $0.545^{1}$ & $48.92^{\mathrm{a}}$ & $46.55^{\mathrm{b}}$ & $0.605^{1}$ & 42.62 & 41.14 & $0.895^{2}$ & $41.45^{\mathrm{a}}$ & $45.26^{\mathrm{b}}$ & $1.070^{12}$ & ns & $* * *$ & * \\
\hline PUFA & $15.56^{\mathrm{a}}$ & $14.33^{\mathrm{b}}$ & $0.267^{1}$ & 13.66 & 13.58 & $0.367^{2}$ & 15.70 & 14.56 & $0.423^{1}$ & 13.61 & 13.33 & $0.341^{2}$ & ns & $* *$ & ns \\
\hline $\mathrm{P} / \mathrm{S}$ & $0.40^{\mathrm{a}}$ & $0.36^{\mathrm{b}}$ & $0.011^{1}$ & 0.37 & 0.34 & $0.014^{12}$ & 0.38 & 0.33 & $0.017^{1}$ & 0.30 & 0.33 & $0.013^{12}$ & $\mathrm{~ns}$ & $*$ & ns \\
\hline
\end{tabular}

SFA: sum of saturated fatty acids; UFA: sum of unsaturated fatty acids; PUFA: sum of polyunsaturated fatty acids; MUFA: sum of monounsaturated fatty acids; P/S: the ratio of total PUFA to total SFA; Fem: female; S: significantly different values as influenced by sex $*(\mathrm{P}<0.05) ; * *(\mathrm{P}<0.01) ; * * *(\mathrm{P}<0.001)$; ns: no significant difference; L: significantly different values as influenced by location $*(\mathrm{P}<0.05) ;{ }^{* *}(\mathrm{P}<0.01) ; * *(\mathrm{P}<0.001)$; SxL: interaction of sex and location $*(\mathrm{P}<0.05) ; * *(\mathrm{P}<0.01) ; * * *(\mathrm{P}<0.001) ;{ }^{\mathrm{a}-\mathrm{b}}$ Means within the same row not followed by the same letter differ significantly (influence of sex $)(\mathrm{P}<0.05) ;{ }^{\mathrm{i}-4} \mathrm{SEM}$ within the same row not followed by the same letter differ significantly (influence of location) $(\mathrm{P}<0.05)$.

\section{DISCUSSION}

The statistical analysis showed some differences in the contents of some fatty acids of the studied locations. However, the fatty acid profile was very similar in the deposits of fat studied. These results are in agreement with previous findings by other authors in different fat locations (Cava et al., 2003; Franco et al., 2006).

The studied Celta pigs were fed with commercial compound feed (Table 1). These have high contents of carbohydrates and low contents of fat. Their fatty acid composition is characterized by high levels of C18:1 c n-9 and C18:2 n-6. The carbohydrates may serve as substrate in the synthesis of fat, synthesizing C16:0. Also, the dietary retinol may have a direct effect on the deposition of fatty acids such as C16:0 and C18:0 (Olivares et al., 2009a). On the other hand, the enzyme $\Delta-9$ desaturase would be very active in Celta hogs, as it is favored by the carbohydrates and the MUFA, which would explain in part the high content of 


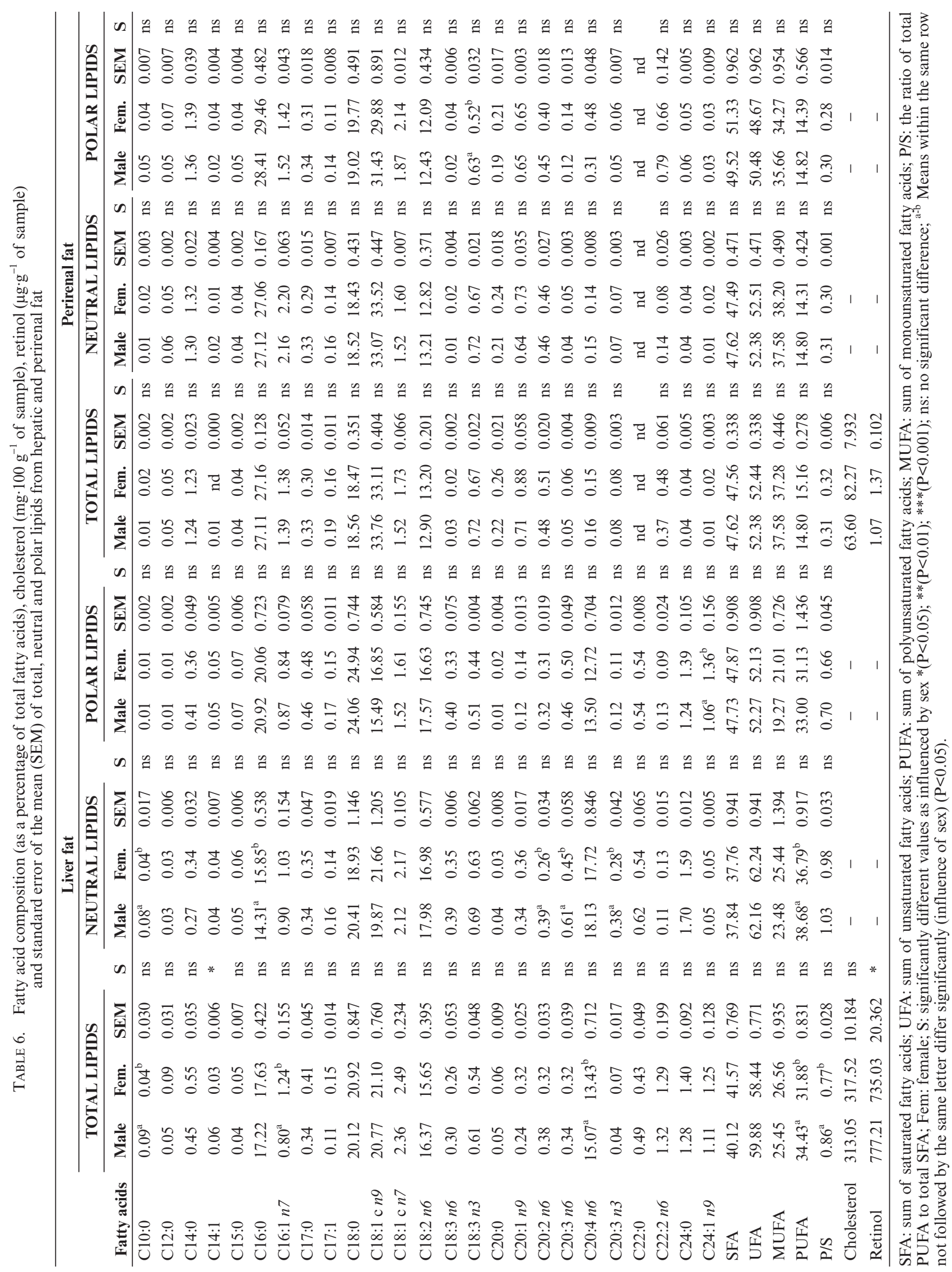




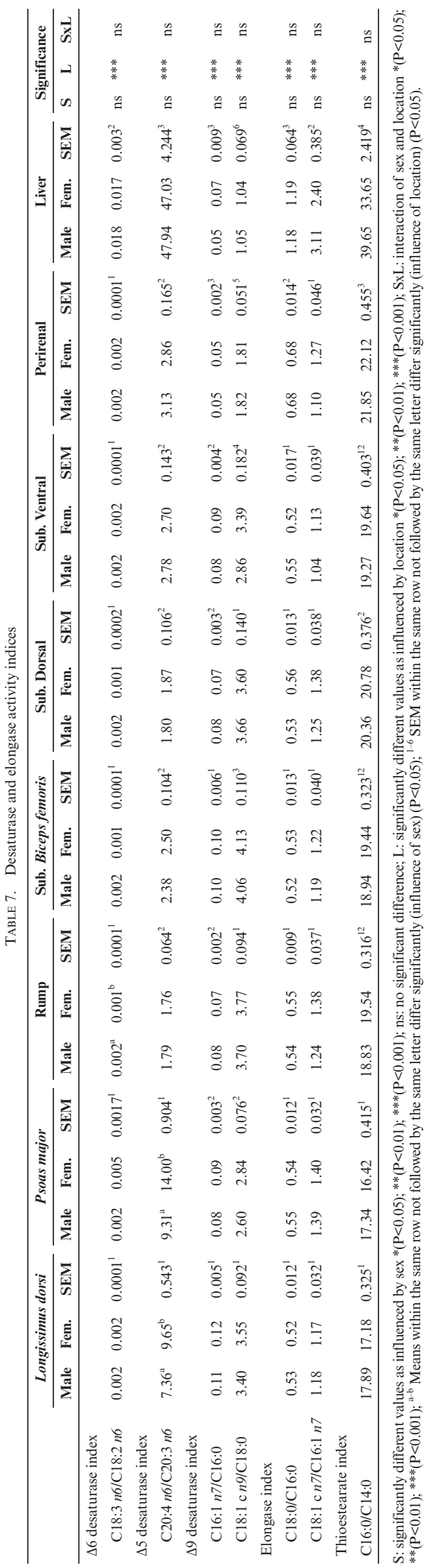

MUFA and especially of C18:1 c n-9 (Franco et al., 2006).

The SFA content was higher in hepatic and perirenal fat than in other deposits. C18:1 c n-9 and UFA contents were higher from intramuscular and subcutaneous fat than for the hepatic and perirenal fat. Oleic acid is synthesized by $\Delta 9$-desaturase (stearoyl-CoA-desaturase). Thompson and Allen (1969) observed that the activity of this enzyme was higher in the subcutaneous adipose tissue than in the perirenal fat, which may explain the higher oleic acid content in the subcutaneous fat.

However, the percentage of C20:4 n- 6 and PUFA content was significantly higher in hepatic fat than in other anatomical locations.

Total lipids and neutral lipids, except for the ones in the liver, showed similar fatty acid composition, as the neutral lipid represented the greatest percentage of total lipids.

The fatty acid composition of total and neutral lipids is more influenced by location than the fatty acids of polar lipids. The proportion of PUFA in the polar lipids from the intramuscular fat was significantly higher than those of the total and neutral lipids. These differences were a consequence of a higher content of some individual fatty acids in the polar lipids such as C18:2 n-6 and C20:4 n-6.

The type of fibers and their cellular metabolism determines the fatty acid composition in the muscle (Andrés et al., 2001; Leseigneur-Meynier and Gandemer, 1991). PM is a predominantly oxidative muscle, showing higher levels of PUFA than LD in both lipid classes, which has been described as a predominantly glucolitic muscle in the scientific literature.

The differences observed in the fatty acids from different locations could also be explained as a function of the amount of neutral lipids and phospholipids that they contain (Cava et al., 2003; Franco et al., 2006).

On the other hand, in monogastric animals such as the pig, the fatty acid composition of different fat deposits is affected by the diet (Viera-Alcaide et al., 2007) and some fatty acids of the diet are absorbed and deposited directly into the fat deposits. Tissue fatty acid composition is not modified to the same extent in the different locations. C18:2 n-6 and C18:3 n-3 are directly accumulated from the diet, while C20:4 n-6 is derived from dietary C18:2 n-6. The studied Celta pigs were fed with commercial feed, which was rich in C18:2 n-6 (Table 1).

The proportion of fatty acids is also determined by endogenous synthesis. Previous studies showed that the activity of the enzymes involved in the lipogenesis is tissue specific (Buller and Enser, 1986; Narváez-Rivas et al., 2009). Our results show that desaturase and elongase activity is significantly different $(\mathrm{P}<0.001)$ in $\mathrm{LD}$ and $\mathrm{PM}$ muscles. 
Traditionally, male and female Celta pigs are castrated to reduce the negative effect of oestrous on growth, to improve meat quality and to facilitate management. The effect of castration on lipid content and fatty acid composition has been previously described (Cai et al., 2010; Högberg et al., 2004; Ntawubizi et al., 2009; Razmaite et al., 2008). In most cases, these studies are carried out on entire males, females or castrated males. However, little is known about the effect of castration on the females.

Our results showed that the sex exerted less influence than the anatomical location on fatty acid contents. However, there were differences in some fatty acids of the intramuscular and subcutaneous fat between castrated females and castrated males. The fatty acids of neutral lipids were also more influenced by sex than the fatty acids of polar lipids.

Results reported in the literature about the effect of sex on fatty acids show that the most important differences between sexes in fatty acid composition are caused by a difference in metabolism between females and males or between castrated and uncastrated pigs. However, most of these sex differences are eliminated by the castration of males and females because the castration causes a removal of hormones that affects the fatty acid metabolism (Högberg, 2002).

In addition, the age of castration has an influence on the effect of sex on meat quality. In the case of castrated animals at a young age, the sexual hormones have little influence on growth (Mayoral et al., 1999). The differences observed in the literature could also, at least in part, be due to the different ages of castration.

Our results showed differences among some fatty acids in castrated females and castrated males. For example, C17:0 in the total lipid contents from the intramuscular fat, $\mathrm{C} 15: 0$ in total lipids and $\mathrm{C} 17: 1$ in polar lipids from the subcutaneous fat showed significant effects of the sex. In contrast to our results, Ramírez and Cava (2008) did not find differences in fatty acid compositions in subcutaneous and intramuscular fat from LD and Biceps femoris muscles between castrated females and castrated males. Serrano et al. (2008) did neither observe any difference in the fatty acid contents of the subcutaneous fat of castrated females and castrated males. Cordero et al. (2010) did not find any difference in the fatty acid composition of the intramuscular fat from LD between castrated females and castrated males in the Iberian $\times$ Duroc pig. However, these authors found differences in C18:1 c $n-9$, SFA and the MUFA of the subcutaneous fat between sexes.

Peinado et al. (2008) did not observe differences between the fatty acids of subcutaneous fat of intact females, castrated females and castrated males, except for the C18:2 n-6 content, which was higher for intact females than for castrated females and castrated males.
On the contrary, a significant effect $(\mathrm{P}<0.001)$ of location and sex was observed for the $\Delta 5$ desaturase index. PM presented a higher $\Delta 5$ desaturase index than LD in females than in males. $\Delta-5$ desaturase activity, which is an indicator of the stearoyl-CoA desaturase influence on the conversion of C20:3 n-6 to $C 20: 4 n-6$, was higher in castrated females than in castrated males.

Hasty et al. (2002) showed that fatty acid metabolism follows a different course according to sex. Cordero et al. (2010) observed that the subcutaneous fat from castrated males had lower MUFA/ SFA and C18:1 c $n-9 / C 18: 0$ ratios than those from castrated females. However, Zhang et al. (2007) did not observe any differences in the indices of $\Delta 9$-desaturase, thioesterase, or elongase between males and females.

The cholesterol contents of muscles coincided with those described by Bragagnolo and RodríguezAmaya (2002) and Salvatori et al. (2008). The fact that the PM muscle presented a higher cholesterol content $(P>0.05)$ than the LD muscle could be due to that oxidative muscles are richer in phospholipids than glycolytic muscles, and as a result, they are richer in cholesterol (Alasnier et al., 1996; Chizzolini et al., 1999).

Intramuscular fat content also affects the amount of cholesterol, since the muscles with high content of intramuscular fat present proportionately less membrane polar lipids and therefore lower amounts of the cholesterol associated with these membranes (Alasnier et al., 1996). This would explain, in part, the fact that the LD muscle has a lower cholesterol content than the PM muscle.

Bragagnolo and Rodríguez-Amaya (2002) found that cholesterol contents decreased significantly with increasing age at slaughter. In turn, Salvatori et al. (2008) found a significant relationship between carcass weight and cholesterol content, the last one decreased as the weight of the carcass increased. This may explain, in part, the low-cholesterol in Celta pig muscles, as these pigs were slaughtered at an older age (16 months), presenting a high carcass weight and high percentage of intramuscular fat. So, despite not being significant, we found that there was an inverse correlation between carcass weight and cholesterol content.

Several authors suggest that castration causes increased intramuscular fat deposition (Latorre et al., 2003; Mersmann, 1984; Mourot et al., 1999; Peinado et al., 2008), because the pigs have greater energy, resulting in an increased fat storage (Gandemer et al., 1979; Kolstad et al., 1996). These differences were not noted in our study, as both males and females were castrated.

There are few studies that describe the content of retinol in pig muscles. Högberg et al. (2002) and Olivares et al. (2009a) described a retinol content of approximately 0.03 and $0.04 \mu \mathrm{g} \cdot \mathrm{g}^{-1}$ of pork loin 
samples, respectively, showing that the retinol content from muscles is very low.

The retinol and cholesterol contents from hepatic fat were much higher than those observed in other locations. This is mainly due to the fact that liver synthesized and accumulated these substances, which explains their high contents in relation with the other locations. The retinol content present in the Celta pork liver was above the one described by Olivares et al. (2009a, 2009b) possibly due to a higher content of vitamin $\mathrm{A}$ in the diet of the Celta pig (Table 1).

The percentage of intramuscular fat and cholesterol contents in muscles and the retinol and cholesterol contents in the subcutaneous and perirrenal fat showed no significant differences between males and females. However, there appears to be a greater deposition $(\mathrm{P}<0.05)$ of retinol in the liver of males compared to females.

The absence of significant differences in the content of cholesterol and retinol due to sex can be due to the fact that castration removed hormones that affect cholesterol and retinol metabolism and, as noted above, differentiate males from females.

\section{CONCLUSIONS}

The effects of gender and location on the fatty acid profile were less intense in polar lipids than in total and neutral lipids. The differences determined by the location could be due to the different activity of the elongase and desaturase enzymes. In turn, the cholesterol content decreased with increasing age of slaughter, weight, and amount of intramuscular fat.

Castration removed hormones that differentiate sexes, so as both male and female pigs were castrated, only significant differences could be seen in some minor fatty acids. Cholesterol, retinol and intramuscular fat contents showed no significant differences between sexes.

\section{ACKNOWLEDGEMENT}

This work was financially supported by the Spanish Ministry of Science and Innovation (Grant AGL2008-0574-C02-01/ALI). The authors also wish to thank to the "Instituto Orensano de Desarrollo (INORDE)" for its valuable collaboration in the rearing of the pigs.

\section{REFERENCES}

Alasnier C, Rémignon H, Gandemer G. 1996. Lipid characteristics associated with oxidative and glycolitic fibres in rabbit muscles. Meat Sci. 43, 213-224. http://dx.doi.org/10.1016/ S0309-1740(96)00015-0

Andrés AI, Cava R, Mayoral AI, Tejeda JF, Morcuende D, Ruíz J. 2001. Oxidative stability and fatty acid composition of pig muscles as affected by rearing system, crossbreeding and metabolic type of muscle fibre. Meat Sci. 59, 39-47. http://dx.doi.org/10.1016/S0309-1740(01)00050-X.
Association of Official Analytical Chemist. 2000. Official methods of analysis, 17th edition. AOAC, Arlington, VA, USA.

Bermúdez R, Franco I, Franco D, Carballo J, Lorenzo JM. 2012. Influence of inclusion of chestnut in the finishing diet on fatty acid profile of dry-cured ham from Celta pig breed. Meat Sci. 92, 394-399. http://dx.doi.org/10.1016/j. meatsci.2012.05.001

Bermúdez R, Franco D, Carballo J, Sentandreu MA, Lorenzo JM. 2014. Influence of muscle type on the evolution of free amino acids and sarcoplasmic and myofibrillar proteins through the manufacturing process of Celta drycured ham. Food Res. Int. 56, 226-235. http://dx.doi. org/10.1016/j.foodres.2013.12.023.

Bragagnolo N, Rodríguez-Amaya DB. 2002. Simultaneous determination of total lipid, cholesterol and fatty acids in meat and backfat of suckling and adult pigs. Food Chem. 79, 255 260. http://dx.doi.org/10.1016/S0308-8146(02)00136-X.

Buller KJ, Enser M. 1986. The effect of food intake and dietary fatty acids on the activity of stearoyl-CoA $\Delta 9$ desaturase in pig adipose tissue. J. Agric. Sci. 106, 601-609. http:// dx.doi.org/10.1017/S0021859600063474.

Cai ZW, Zhao WF, Jiang XL, Yao YC, Zhao CJ, Xu NY, Wu CX. 2010. Comparison of muscle amino acid and fatty acid composition of castrated and uncastrated male pigs at different slaughter ages. Ital. J. Anim. Sci. 9, 173-178.

Carril J, Rivero G, García J, Rivero C, Vázquez J, Fernández M. 2001. Celta pig breed presentation. Arch. Zootec. 50, 291-299.

Cava R, Estévez M, Ruíz J, Morcuende D. 2003. Physicochemical characteristics of three muscles from free-range reared Iberian pigs slaughtered at $90 \mathrm{~kg}$ live weight. Meat Sci. 63, 533-541. http://dx.doi.org/10.1016/S0309-1740(02) 00180-8.

Chizzolini R, Zanardi E, Dorigoni V, Ghidini S. 1999. Calorific value and cholesterol content of normal and low-fat meat and meat products. Trends Food Sci. Tech. 10, 119-128. http://dx.doi.org/10.1016/S0924-2244(99)00034-5.

Cordero G, Isabel B, Menoyo D, Daza A, Morales J, Piñeiro C, López-Bote CJ. 2010. Dietary CLA supplementation and gender modify fatty acid composition of subcutaneous and intramuscular fat in Iberian x Duroc finishing heavy pigs. Span. J. Agr. Res. 8, 962-970.

De Pedro E, Casillas M, Miranda CM 1997. Microwave oven application in the extraction of fat from the tissue of Iberian pig ham. Meat Sci. 45, 45-51. http://dx.doi. org/10.1016/S0309-1740(96)00097-6.

Delgado GL, Gómez CS, Rubio LMS, Capella VS, Méndez MD, Labastida RC. 2002. Fatty acid and triglyceride profiles of intramuscular and subcutaneous fat from fresh and dry-cured hams from Hairless Mexican pigs. Meat Sci. 61, 61-65. http://dx.doi.org/10.1016/S0309-1740(01) 00163-2.

Folch J, Lees M, Sloane-Stanley GH. 1957. A simple method for the isolation and purification of total lipids from animal tissues. J. Biol. Chem. 226, 497-509.

Franco I, Escamilla MC, García J, García-Fontán MC, Carballo J. 2006. Fatty acid profile of the fat from Celta pig breed fattened using a tradicional feed. Effect of the location in the carcass. J. Food Compos. Anal. 19, 792-799. http:// dx.doi.org/10.1016/j.jfca.2006.05.005.

Gandemer G, Pascal G, Durand G. 1979. Developmental changes in lipogenic enzyme activities in liver and adipose tissue of post-weaning rats. Effect of sex and castration. Ann. Biol. Anim. Biochem. Biophys. 19, 573-581. http:// dx.doi.org/10.1051/rnd:19790503.

Gandemer G, Viau M, Caritez JC, Legault C. 1992. Lipid composition of adipose tissue and muscle in pigs with an increasing proportion of Meishan genes. Meat Sci. 23, 105-121. http://dx.doi.org/10.1016/0309-1740(92)90020-5.

García-Olmo J, De Pedro E, Garrido A, Paredes A, Sanabria C, Santolalla M, Salas J, García-Hierro JR, González I, García Cachan MD, Guirao J. 2002. Determination of the precision of the fatty acid analysis of Iberian pig fat by gas chromatography. Results of a mini collaborative study. Meat Sci. 60, 103-109. http://dx.doi.org/10.1016/ S0309-1740(01)00166-8. 
Gómez M, Lorenzo JM. 2013. Effect of fat level on physicochemical, volatile compounds and sensory characteristics of dry-ripened "chorizo" from Celta pig breed. Meat Sci. 95, 658-666. http://dx.doi.org/10.1016/j.meatsci.2013.06.005.

Hasty JL, Van Heugten E, See MT, Larick DK. 2002. Effect of vitamin $\mathrm{E}$ on improving pork quality in Berkshire and Hampshire sired pigs. J. Anim. Sci. 80, 3230-3237.

Högberg A. 2002. Fatty acids, tocopherols and lipid oxidation in pig muscle. Effects of feed, sex and outdoor rearing. Acta Universitatis Agriculturae Suecia, Agraria 328. Sweden: Swedish University of Agricultural Sciences, Department of Food Science Uppsala.

Högberg A, Pickova J, Babol J, Andersson K, Dutta PC. 2002. Muscle lipids, vitamins $\mathrm{E}$ and $\mathrm{A}$, and lipid oxidation as affected by diet and RN genotype in female and castrated male Hampshire crossbreed pigs. Meat Sci. 60, 411-420. http://dx.doi.org/10.1016/S0309-1740(01)00153-X.

Högberg A, Pickova J, Stern S, Lundström K, Bylund AC. 2004. Fatty acid composition and tocopherol concentrations in muscle of entire male, castrated male and female pigs, reared in an indoor or outdoor housing system. Meat Sci. 68, 659-665. http://dx.doi.org/10.1016/j.meatsci.2004.06.001.

ISO (International Organization for Standarization). 1979. Meat and meat content. Determination of fat, ISO 1443:1979 standard. In: International standards meat and meat products. Geneva, Switzerland.

Kaluzny MA, Duncan LA, Merritt MV, Epps DE. 1985. Rapid separation of lipids classes in high yield and purity using bonded phase columns. J. Lipid Res. 26, 135-140.

Kolstad K, Jopson NB, Vangen O. 1996. Breed and sex differences in fat distribution and mobilization in growing pigs fed at maintenance. Livest. Prod. Sci. 47, 33-41. http:// dx.doi.org/10.1016/S0301-6226(96)01001-9.

Latorre MA, Lázaro R, Gracia MI, Nieto M, Mateos GG. 2003. Effect of sex and terminal sire genotype on performance, carcass characteristics and meat quality of pigs slaughtered at $117 \mathrm{~kg}$ body weight. Meat Sci. 65, 1369-1377. http://dx.doi.org/10.1016/S0309-1740(03)00059-7.

Leseigneur-Meynier A, Gandemer G. 1991. Lipid composition of pork muscle in relation to the metabolic type of the fibres. Meat Sci. 29, 229-241. http://dx.doi.org/ 10.1016/0309-1740(91)90052-R.

López-Cervantes J, Sánchez-Machado DI, Ríos-Vázquez NJ. 2006. High-performance liquid chromatography method for the simultaneous quantification of retinol, $\alpha$-tocopherol, and cholesterol in shrimp waste hydrolysate. J. Chromatogr. 1105, 135-139. http://dx.doi.org/10.1016/j.chroma.2005.08.010.

Lorenzo JM, Carballo J, Franco D. 2013. Effect of the inclusion of chestnut in the finishing diet on volatile compounds of dry-cured ham from Celta pig breed. J. Integr. Agr. 12, 2002 2012. http://dx.doi.org/10.1016/S2095-3119(13)60638-3

Lorenzo JM, Franco D, Carballo J. 2014. Effect of the inclusion of chestnut in the finishing diet on volatile compounds during the manufacture of dry-cured "Lacón" from Celta pig breed. Meat Sci. 96, 211-223. http://dx.doi. org/10.1016/j.meatsci.2013.07.007

Mayoral AI, Dorado M, Guillén MT, Robina A, Vivo JM, Vázquez C, Ruíz J. 1999. Development of meat and carcass quality characteristics in Iberian pigs reared outdoors. Meat Sci. 52, 315-324. http://dx.doi.org/10.1016/ S0309-1740(99)00008-X

Mersmann HJ. 1984. Adrenergic control of lipolysis in swine adipose tissue. Comp. Biochem. Physiol. B 77, 43-53.

Mourot J, Kouba M, Salvatori G. 1999. Facteurs de variation de la lipogenèse dans les adipocytes et les tissus adipeux chez le porc. INRA Productions Animales 12, 311-318.

Narváez-Rivas M, León-Camacho M. Vicario IM. 2009. Fatty acid triacylglycerol composition of the subcutaneous fat from Iberian pigs fattened on the traditional feed: "Montanera" effect of anatomical location and length of feeding. Grasas Aceites 60, 238-247. http://dx.doi.org/10.3989/gya.130308.

Ntawubizi M, Raes K, Buys N, De Smet S. 2009. Effect of sire and sex on the intramuscular fatty acid profile and indices for enzyme activities in pigs. Livest. Sci. 122, 264-270. http://dx.doi.org/10.1016/j.livsci.2008.09.008.
Olivares A, Daza A, Rey AI, López-Bote CJ. 2009a. Interactions between genotype, dietary fat saturation and vitamin A concentration on intramuscular fat content and fatty acid composition in pigs. Meat Sci. 82, 6-12. http://dx.doi. org/10.1016/j.meatsci.2008.11.006.

Olivares A, Daza A, Rey AI, López-Bote CJ. 2009b. Dietary vitamin A concentration alters fatty acid composition in pigs. Meat Sci. 81, 295-299. http://dx.doi.org/10.1016/j. meatsci.2008.07.029

Palanska O, Hetenyi L, Ondrejicka R, Mojto J, Kmetova E. 1993. Fatty acid composition of intramuscular fat of the Longissimus lumborum et thoracis in pigs of various slaughter weights. Zivocisna Vyrova 38, 377-384.

Peinado J, Medel P, Fuentetaja A, Mateos GG. 2008. Influence of castration of females on growth performance and carcass and meat quality of heavy pigs destined to the drycured industry. J. Anim. Sci. 86, 1410-1417. http://dx.doi. org/10.2527/jas.2006-807.

Ramírez MR, Cava R. 2008. Effect of Iberian x Duroc genotype on composition and sensory properties of dry-cured ham. J. Sci. Food Agric. 88, 667-675. http://dx.doi.org/10.1002/ jsfa.3133.

Razmaite V, Kerziene S, Siukscius A. 2008. Pork fat composition of male hybrids from Lithuanian indigenous wattle pigs and wild boar intercross. Food Sci. Tech. Int. 14, 251-257. http://dx.doi.org/10.1177/1082013208094120.

Rentfrow G, Sauber TE, Allee GL, Berg EP. 2003. The influence of diets containing either conventional corn, conventional corn with choice white grease, high oil corn, or high oil high oleic corn on belly/ bacon quality. Meat Sci. 64, 459466. http://dx.doi.org/10.1016/S0309-1740(02)00215-2.

Saldanha T, Frankland ACH, Nogueira M, Bragagnolo N. 2006. HPLC separation and determination of 12 cholesterol oxidation products in fish: comparative study of RI, UV and APCI-MS detectors. J. Agric. Food. Chem. 54, 4107-4113. http://dx.doi.org/10.1021/jf0532009.

Salvatori G, Filetti F, Di Cesare C, Maiorano G, Pilla F, Oriani G. 2008. Lipid composition of meat and backfat from Casertana purebred and crossbred pigs reared outdoors. Meat Sci. 80, 623-631. http://dx.doi.org/10.1016/j. meatsci.2008.02.013

Serrano MP, Valencia DG, Fuentetaja A, Lázaro R, Mateos GG. 2008. Effect of gender and castration of females and slaughter weight on performance and carcass and meat quality of Iberian pigs reared under intensive management systems. Meat Sci. 80, 1122-1128. http://dx.doi. org/10.1016/j.meatsci.2008.05.005.

Thompson EH, Allen E. 1969. Relationship between stearic acid desaturase and fatty acid composition. J. Anim. Sci. 29, $127-128$

Václavková E, Bečková R. 2007. Effect of linseed in pig diet on meat quality and fatty acid content. Archiv Tierzucht 50, $144-151$.

Vázquez C, Menaya C, Benito J, Ferrera JL, Fallola A, García Casco JM. 1996. Perfil de ácidos grasos en distintas localizaciones del tejido adiposo del cerdo ibérico. Eurocarne 52, $17-22$.

Viera-Alcaide I, Vicario IM, Graciani Constante, E, LeónCamacho M. 2007. Authentication of fattening diet of Iberian pig according to their triacylglycerols profile from subcutaneous fat. Anal. Chim. Acta 596, 319-324. http:// dx.doi.org/10.1016/j.aca.2007.06.026.

Wood JD, Enser M, Whittington FM, Moncrieff CB, Kemster AJ. 1989. Backfat composition in pigs: differences between fat thickness groups and sexes. Livest. Prod. Sci. 22, 351362. http://dx.doi.org/10.1016/0301-6226(89)90066-3.

Wood JD, Richardson RI, Nute GR, Fisher AV, Campo MM, Kasapidou E, Sheard PR, Enser M. 2004. Effects of fatty acids on meat quality: a review. Meat Sci. 66, 21-32. http:// dx.doi.org/10.1016/S0309-1740(03)00022-6.

Zhang S, Knight TJ, Stalder KJ, Goodwin RN, Lonergan SM, Beitz DC. 2007. Effects of breed, sex, and halothane genotype on fatty acid composition of pork longissimus muscle. J. Anim. Sci. 85, 583-591. http://dx.doi.org/10.2527/ jas.2006-239. 